\title{
A Physicist View of COVID-19 Airborne Infection through Convective Airflow in Indoor Spaces
}

\author{
Luis A. Anchordoqui ${ }^{a^{*}}$, Eugene M. Chudnovsky ${ }^{\text {a }}$ \\ ${ }^{a}$ Physics Department, Herbert H. Lehman College and Graduate School, The City University of New York 250 Bedford Park Boulevard \\ West, Bronx, New York 10468-1589, United States.
}

Received 02 August 2020; Accepted 25 August 2020

\begin{abstract}
General Idea: Naturally produced droplets from humans (such as those produced by breathing, talking, sneezing, and coughing) include several types of cells (e.g., epithelial cells and cells of the immune system), physiological electrolytes contained in mucous and saliva (e.g. $\mathrm{Na}+, \mathrm{K}+, \mathrm{Cl}-$ ), as well as, potentially, several infectious agents (e.g. bacteria, fungi, and viruses). In response to the novel coronavirus SARS-CoV-2 epidemic, which has become a major public health issue worldwide, we provide a concise overview of airborne germ transmission as seen from a physics perspective. We also study whether coronavirus aerosols can travel far from the immediate neighbourhood and get airborne with the convective currents developed within confined spaces. Methodology: Methods of fluid dynamics are utilized to analyse the behavior of various-size airborne droplets containing the virus. Study Findings: We show that existing vortices in the air can make a location far away from the source of the virus be more dangerous than a nearby (e.g., 6 feet away) location. Practical Implications: Our study reveals that it seems reasonable to adopt additional infection-control measures to the recommended 6 feet social distancing. We provide a recommendation that could help to slow down the spread of the virus.
\end{abstract}

Keywords: COVID-19; Novel Coronavirus; Airborne Infection; Indoor Spaces.

\section{Introduction}

The recent outbreak of the respiratory disease identified as COVID-19 is caused by the severe acute respiratory syndrome coronavirus 2, shortened to SARS-CoV- 2 [1-3]. The outbreak, first reported in December 2019, has rapidly evolved into a global pandemic. Indeed, COVID-19 is spreading across the globe with a speed and strength that laid bare the limits of our understanding of the transmission pathways and the associated factors that are key to the spread of such diseases. In particular, the virus can spread from seemingly healthy carriers or people who had not yet developed symptoms [4]. Overall, this has transformed the face of healthcare around the world.

To understand and prevent the spread of a virus like SARS-CoV-2, it is important to estimate the probability of airborne transmission as aerosolization with particles potentially containing the virus. There have been reports favoring the possibility of creating coronavirus aerosols [5]. Thus far no aerosolized coronavirus particles have been found in hospital searches within the most public areas, but evidence has been detected in rooms of SARS-CoV-2 patients [6-9]. In this paper we provide an overview on the possible threat of SARS-CoV-2 airborne infection from a physics point of view, focusing attention on the effect of convection currents in indoor spaces.

The layout of the paper is as follows. In Sec. 2 we first provide a concise discussion of the motion of the virus in suspended aerosols. After that, using the SimScale program [10] we study the convective airflow in a meeting room

* Corresponding author: luis.anchordoqui@gmail.com

$>$ This is an open access article under the CC-BY license (https://creativecommons.org/licenses/by/4.0/).

(C) Authors retain all copyrights. 
and office space. We show that existing vortices in the air can make a location far away from the source of the virus be more dangerous than nearby (e.g., 6 feet away) location. In Sec. 3 we present our conclusions. Throughout we adopt the convention of the World Health Organization to nickname particles that are $\geq 5 \mu \mathrm{m}$ diameter as droplets and those $\leq 5 \mu \mathrm{m}$ as aerosols or droplet nuclei [11].

\section{Modeling the Effect of Convection Currents in the Transmission of SARS-COV-2}

In the presence of air resistance, compact heavy objects fall to the ground quickly, while light objects exhibit Brownian motion and follow the pattern of turbulent convection of the air. For aerosol particles containing the virus, the boundary between these two behaviors depends on the size of the particle. We begin with a simple question: how long does a virus float in the air under the influence of gravity? To answer this query we model the virus as a sphere of radius $\mathrm{r} \sim 90 \mathrm{~nm}$ and mass $\mathrm{m} \sim 2.5 \times 10^{-19} \mathrm{~kg}$ [12], and we assume that this spherical particle is suspended in a viscous fluid (the air) feeling the Earth's gravitational field. Herein, gravity tends to make the particles settle, while diffusion and convection act to homogenize them, driving them into regions of smaller concentration. On the one hand, the convection mechanism provides particle macro- mixing within the fluid through the tendency of hotter and consequently less dense material to rise, and colder, denser material to sink under the influence of gravity. On the other hand, the diffusion mechanism acts on the scale of an individual particle (micro-mixing) slowly and randomly moving through the media.

Under the action of gravity, the virus acquires a downward terminal speed that follows from Stokes law and is given by:

$v_{\text {down }}=\mu m g$

Where $\mathrm{g}=9.8 \mathrm{~m} / \mathrm{s}^{2}$ is the acceleration due to gravity and;

$\mu=\frac{1}{6 \pi \eta r}$

Is the virus mobility in the fluid, and where $\eta=1.8 \times 10^{-5} \mathrm{~kg} /(\mathrm{m} \mathrm{s})$ is the dynamic viscosity of air [13]. Substituting (2) into (1) we find that the downward terminal speed of the virus in dry air is indeed negligible, $v_{\text {down }} \sim 8 \times 10^{-8} \mathrm{~m} / \mathrm{s}$. It is therefore clear that gravity plays no role in the motion of an isolated virus through the air. Rather it follows a convection pattern in a manner similar to how smelly substances move through the air. The survival probability of the virus in the dry air is then given by the likelihood of survival outside its natural environment. The half-life of SARSCoV-2 in aerosols has been found to be about 1.1 hours [5].

We have seen that the coronavirus can go airborne staying suspended in the air. However, the virus is transmitted through respiratory droplets and droplet nuclei produced mostly while sneezing and coughing. Then to ascertain whether airborne transmissible SARS-CoV-2 can survive and stay infectious in aerosols we must double-check that the falling time of a droplet or droplet nuclei from a height of about two meters is larger than its evaporation time scale. To this end, we assume that the drops are also spherical and hence the mass can be simply estimated as:

$m=\frac{4}{3} \pi r^{3} \rho$

Where $\rho=997 \mathrm{~kg} / \mathrm{m}^{3}$ is the density of water and $\mathrm{r}$ the droplet/aerosol radius. For large droplets whose diameters $\geq 1000 \mu \mathrm{m}$, the effect of air resistance is negligible and so the falling time can be directly estimated using Newton's equations for gravitational settling. For smaller droplets whose diameters $<100 \mu \mathrm{m}$, the falling times must instead be determined using the downward terminal speed given in (1) to account for the air resistance upon the falling droplets. It is now an instructive and straightforward exercise to show that the time for falling $2 \mathrm{~m}$ in saturated air is $0.6 \mathrm{~s}$ for droplets with $\mathrm{r}>500 \mu \mathrm{m}, 6.0 \mathrm{~s}$ for those of $\mathrm{r} \sim 50 \mu \mathrm{m}, 600 \mathrm{~s}$ (about 10 minutes) for those of $\mathrm{r} \sim 5 \mu \mathrm{m}$, and $60,000 \mathrm{~s}$ (about 16.6 hours) for those of $\mathrm{r} \sim 0.5 \mu \mathrm{m}$. The droplet evaporation time scale, as computed by Wells using droplet evaporation data collected by Whytlaw-Gray and Patterson, is shown in Table 1 [14]. The assumption of pure water droplets in unsaturated air at $18^{\circ} \mathrm{C}$ was used for the evaporation calculations, such that the theoretical droplets are capable of complete evaporation. By direct comparison of the droplet evaporation and falling times we can conclude that somewhere between 100 and $200 \mu \mathrm{m}$ lies the droplet size (i.e. the diameter) which identifies droplets of mouth spray that reach the ground within the life of the droplet as against droplets that evaporate and remain in the air as droplet-nuclei with attached SARS-CoV-2 infection. Several investigations have been carried out to continue improving the precision of Wells analyses and to study the various external environmental (such as temperature and humidity) factors that may alter his estimates; see e.g. [15]. 
Table 1. Evaporation Time of Water Droplets

\begin{tabular}{cc}
\hline Droplet diameter $(\boldsymbol{\mu m})$ & Evaporation time $(\mathbf{s})$ \\
\hline 2000 & 660 \\
1000 & 165 \\
500 & 41 \\
200 & 6.6 \\
100 & 1.7 \\
50 & 0.4 \\
\hline
\end{tabular}

The sizes of the droplets and droplet-nuclei produced by sneezing and coughing were studied by the microscopic measurement of 12,000 droplet stain-marks found on slides exposed directly to mouth-spray, and of 21,000 staincontaining droplet-nuclei recovered from the air on to oiled slides exposed in the slit sampler [16]. From this data sample it was found that the original diameters of respiratory droplets ranged from 1 to $2000 \mu \mathrm{m}$ and that $95 \%$ were between 2 and $100 \mu \mathrm{m}$ and that the most common were between 4 and $8 \mu \mathrm{m}$. Similar results were reported in Gralton et al. (2011) [17]. This suggests that, in principle, droplet-spray could drive direct airborne infection of SARS-CoV-2. The transmission of the COVID-19 disease, however, still depends on the infectious virus load carried by the droplets, which must be determined experimentally. The number of virions needed for infection is yet unknown, but we can use other viral transmission (e.g., influenza [18-20]) for a template. The spread of a sneeze in the air has been studied by ultrafast imaging at MIT [21-23]. It was found that even the largest droplets from a sneeze can float in the air for up to 10 minutes, which allows them to reach the far end of a large room. This point towards convection in the air being the primary mechanism of the spread of the infection.

From the physics point of view, we cannot find a good justification for a stationary 6-feet separation in a situation when people spend long time together in a room. Small droplets or aerosols containing the virus move in the air via convection. The convection pattern in a room can be very complex; see Figure 1.
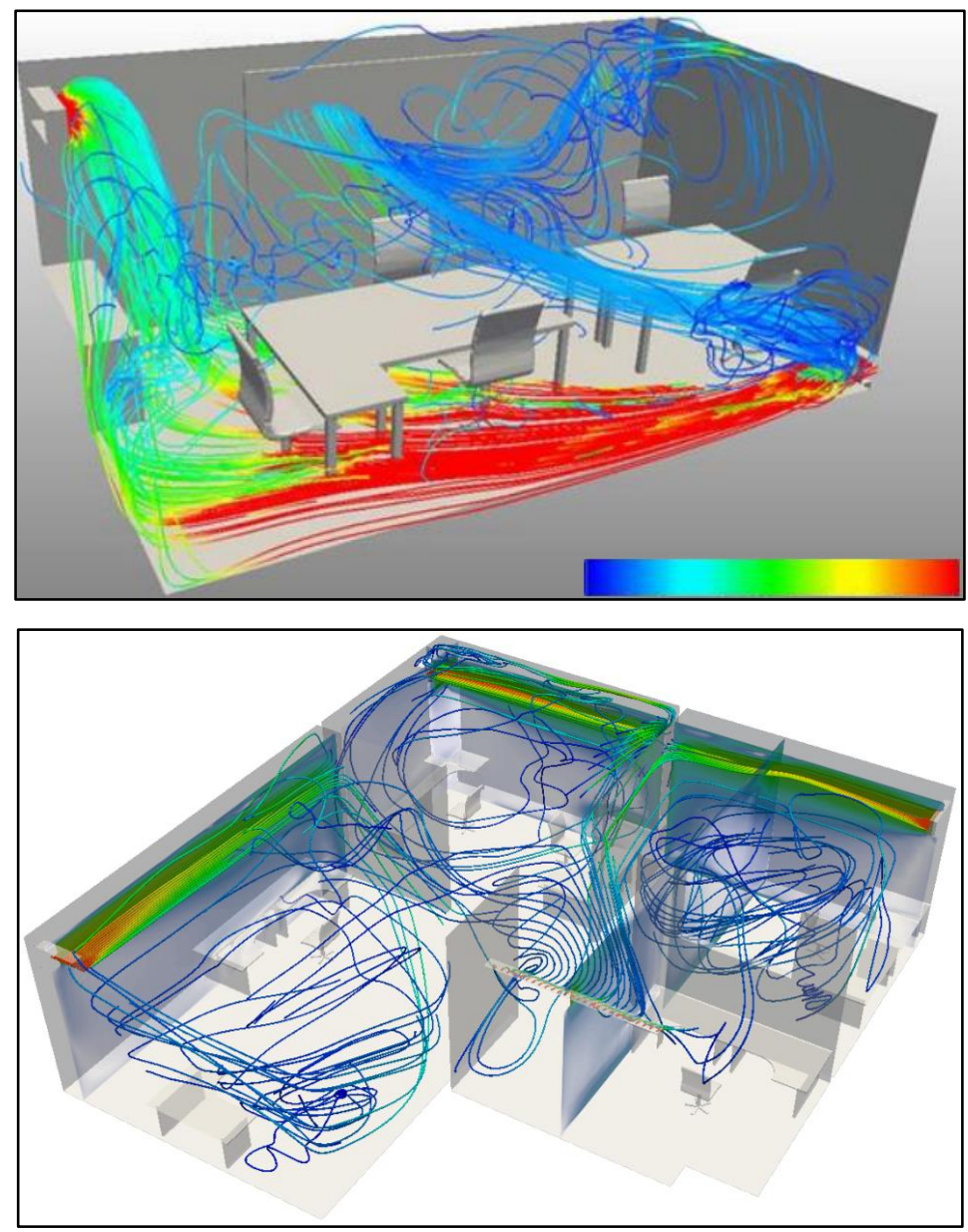

Figure 1. Visual representation of airflow streamlines in a meeting room (left) and office space (right) colored to velocity magnitude from low (blue) to high (red). The convection pattern in the meeting room demonstrates how the infection can be persistently carried by the airflow between two chairs separated by 6 feet. The convection pattern in the office space illustrates how the infection can be taken by the airflow from one cubicle to the other. Simulation by SimScale [10]. 
It depends on the location of air conditioners, radiators, windows, and all items in the room, as well as on people producing vortices by moving around. The existing vortices in the air can make a location far away from the source of the virus more dangerous than the location 6 feet away. This applies to meeting rooms, office spaces, supermarkets, department stores, etc. The airflow pattern should be studied for all such facilities to avoid the spread of infection to large distances from a single infected person. The safest rooms must be those equipped with the air sucking ventilator at the top, like hospital surgery rooms [24].

By all means, re-configuring the ventilation of public and private facilities cannot be done within the timescale of the pandemic. The question is what to do now if we want to slow its pace. The answer is very simple. People must be required to wear face masks in public spaces to prevent the virus from becoming airborne in the first place.

\section{Conclusion}

Airborne SARS-CoV-2 virus spreads from infected individuals and accumulates in confined spaces where it can linger in the air in the aerosol form for hours. The inhaled virus load depends on the virus concentration in the air and the time of exposure. Concentration can vary from one spot to another. It depends on the location of the spreaders and the pattern of the airflow. The latter is determined by many factors, such as the location of doors and windows, ventilators, heaters, movement of people, etc. Common central air conditioning system that is cooling the indoor air but is not exchanging it with the outside air and is not filtering the virus helps to spread it across the air-conditioned space. It can easily take the virus to distant locations and make it accumulate in the least expected places. Computer studies of the airflow in public spaces are important for mitigating this problem. In the long run business and educational facilities should consider redesigning ventilation and air conditioning systems to effectively reduce concentration of the aerosol virus in the air.

\section{Funding}

The theoretical and computational techniques and resources used in this research were supported by the U.S. National Science Foundation, NSF Grant PHY-1620661 (L.A.A.), and the U.S. Department of Energy, Office of Science, DOE Grant DE- FG02-93ER45487 (E.C.).

\section{Role of the Funder/Sponsor}

The sponsors had no role in the preparation, review or approval of the manuscript and decision to submit the manuscript for publication. Any opinions, findings, and conclusions or recommendations expressed in this article are those of the authors and do not necessarily reflect the views of the NSF or DOE.

\section{Declaration of Competing Interest}

The authors declare that they have no known competing financial interests or personal relationships that could have appeared to influence the work reported in this paper.

\section{Ethical Approval}

The manuscript does not contain experiments on animals and humans; hence ethical permission not required.

\section{References}

[1] C. Huang, Y. Wang, X. Li, L. Ren, J. Zhao, Y. Hu, L. Zhang, G. Fan, J. Xu, X. Gu, Z. Cheng, T. Yu, J. Xia, Y. Wei, W. Wu, X. Xie, W. Yin, H. Li, M. Liu, Y. Xiao, H. Gao, L. Guo, J. Xie, G. Wang, R. Jiang, Z. Gao, Q. Jin, J. Wang, and B. Cao, (2020). Clinical features of patients infected with 2019 novel coronavirus in Wuhan, China, The Lancet, 395-497. doi:10.1016/S01406736(20)30183-5.

[2] P. Zhou, X. Yang, X. Wang, B. Hu, L. Zhang, W. Zhang, H. Si, Y. Zhu, B. Li, C. Huang, H. Chen, J. Chen, Y. Luo, H. Guo, R. Jiang, M. Liu, Y. Chen, X. Shen, X. Wang, X. Zheng, K. Zhao, Q. Chen, F. Deng, L. Liu, B. Yan, F. Zhan, Y. Wang, G. Xiao, and Z. Shi, (2020). A pneumonia outbreak associated with a new coronavirus of probable bat origin, Nature, 579(7798), 270273. doi:10.1038/s41586-020-2012-7.

[3] N. Zhu, D. Zhang, W. Wang, X. Li, B. Yang, J. Song, X. Zhao, B. Huang, W. Shi, R. Lu, P. Niu, F. Zhan, X. Ma, D. Wang, W. $\mathrm{Xu}$, G. Wu, G. F. Gao, and W. Tan, (2020). A novel coronavirus from patients with pneumonia in China, 2019, New England Journal of Medicine, 382(8), 727-733. doi:10.1056/nejmoa2001017.

[4] C. Rothe, M. Schunk, P. Sothmann, G. Bretzel, G. Froeschl, C. Wallrauch, T. Zimmer, V. Thiel, C. Janke, W. Guggemos, M. Seilmaier, C. Drosten, P. Vollmar, K. Zwirglmaier, S. Zange, R. W\"olfel, M. Hoelscher, (2020). Transmission of 2019-nCoV Infection from an Asymptomatic Contact in Germany, New England Journal of Medicine, 382(10), 970-971. doi:10.1056/nejmc2001468. 
[5] N. van Doremalen, T. Bushmaker, D. H. Morris, M. G. Holbrook, A. Gamble, B. N. Williamson, A. Tamin, J. L. Harcourt, N. J. Thornburg, S. I. Gerber, J. O. Lloyd-Smith, E. de Wit, and V. J. Munster, (2020). Aerosol and surface stability of SARS-CoV-2 as compared with SARS-CoV-1, New England Journal of Medicine, 382(16), 1564-1567. doi:10.1056/nejmc2004973.

[6] S. W. X. Ong, Y. K. Tan, P. Y. Chia, T. H. Lee, O. T. Ng, M. S. Y. Wong, and K. Marimuthu, (2020). Air, surface environmental, and personal protective equipment contamination by severe acute respiratory syndrome coronavirus 2 (SARSCoV-2) from a symptomatic patient, JAMA, 323(16), 1610. doi:10.1001/jama.2020.322.

[7] J. L. Santarpia, D. N. Rivera, V. Herrera, M. J. Morwitzer, H. Creager, G. W. Santarpia, K. K. Crown, D. M. Brett-Major, E. Schnaubelt, M. J. Broadhurst, J. V. Lawler, St. P. Reid, and J. J. Lowe, (2020). Transmission potential of SARS-CoV-2 in viral shedding observed at the University of Nebraska Medical Center, medRxiv preprint doi:10.1101/2020.03.23.20039446.

[8] Y. Liu, Z. Ning, Y. Chen, M. Guo, Y. Liu, N. K. Gali, L. Sun, Y. Duan, J. Cai, D. Westerdahl, X. Liu, K. Xu, K.-f. Ho, H. Kan, Q. Fu, and K. Lan, (2020). Aerodynamic analysis of SARS-CoV-2 in two Wuhan hospitals, Nature, 582(7813), 557-560. doi:10.1038/s41586-020-2271-3.

[9] Z.-D. Guo, Z.-Y. Wang, S.-F. Zhang, X. Li, L. Li, C. Li, Y. Cui, R.-B. Fu, Y.-Z. Dong, X.-Y. Chi, M.-Y. Zhang, K. Liu, C. Cao, B. Liu, K. Zhang, Y.-W. Gao, B. Lu, and W. Chen, (2020). Aerosol and surface distribution of severe acute respiratory syndrome coronavirus 2 in hospital wards, Wuhan, China, Emerging Infectious Diseases, 26(7), $1583-1591$. doi:10.3201/eid2607.200885.

[10] SimScale - CFD, FEA, Thermal Simulation and CAE, Available Online: https://www.simscale.com (Accessed on 25 August 2020).

[11] Shiu, E. Y. C., Leung, N. H. L., \& Cowling, B. J. (2019). Controversy around airborne versus droplet transmission of respiratory viruses. Current Opinion in Infectious Diseases, 32(4), 372-379. doi:10.1097/qco.0000000000000563.

[12] Woo, P. C. Y., Huang, Y., Lau, S. K. P., \& Yuen, K.-Y. (2010). Coronavirus Genomics and Bioinformatics Analysis. Viruses, 2(8), 1804-1820. doi:10.3390/v2081803.

[13] Einarsson, J., \& Mehlig, B. (2017). Spherical particle sedimenting in weakly viscoelastic shear flow. Physical Review Fluids, 2(6). doi:10.1103/physrevfluids.2.063301.

[14] Wells, W. F. (1934). On Air-Borne Infection: Study Ii. Droplets and Droplet Nuclei. American journal of Epidemiology, 20(3), 611-618.

[15] Liu, F., Qian, H., Zheng, X., Song, J., Cao, G., \& Liu, Z. (2019). Evaporation and dispersion of exhaled droplets in stratified environment. IOP Conference Series: Materials Science and Engineering, 609, 042059. doi:10.1088/1757-899x/609/4/042059.

[16] Duguid, J. P. “The Size and the Duration of Air-Carriage of Respiratory Droplets and Droplet-Nuclei." Epidemiology and Infection 44, no. 6 (September 1946): 471-479. doi:10.1017/s0022172400019288.

[17] Gralton, J., Tovey, E., McLaws, M.-L., \& Rawlinson, W. D. (2011). The role of particle size in aerosolised pathogen transmission: A review. Journal of Infection, 62(1), 1-13. doi:10.1016/j.jinf.2010.11.010.

[18] Yang, W., Elankumaran, S., \& Marr, L. C. (2011). Concentrations and size distributions of airborne influenza A viruses measured indoors at a health centre, a day-care centre and on aeroplanes. Journal of the Royal Society Interface, 8(61), 11761184. doi:10.1098/rsif.2010.0686.

[19] Yezli, S., \& Otter, J. A. (2011). Minimum Infective Dose of the Major Human Respiratory and Enteric Viruses Transmitted Through Food and the Environment. Food and Environmental Virology, 3(1), 1-30. doi:10.1007/s12560-011-9056-7.

[20] Nikitin, N., Petrova, E., Trifonova, E., \& Karpova, O. (2014). Influenza Virus Aerosols in the Air and Their Infectiousness. Advances in Virology, 2014, 1-6. doi:10.1155/2014/859090.

[21] Bourouiba, L., Dehandschoewercker, E., \& Bush, J. W. M. (2014). Violent expiratory events: on coughing and sneezing. Journal of Fluid Mechanics, 745, 537-563. doi:10.1017/jfm.2014.88.

[22] Scharfman, B. E., Techet, A. H., Bush, J. W. M., \& Bourouiba, L. (2016). Visualization of sneeze ejecta: steps of fluid fragmentation leading to respiratory droplets. Experiments in Fluids, 57(2). doi:10.1007/s00348-015-2078-4.

[23] Bourouiba L., (2020). Turbulent gas clouds and respiratory pathogen emissions: Potential implications for reducing transmission of COVID-19, JAMA 323, 1837. doi:10.1001/jama.2020.4756.

[24] H. C. Yu, K. W. Mui, L. T. Wong, and H. S. Chu, (2016). Ventilation of general hospital wards for mitigating infection risks of three kinds of viruses including Middle East respiratory syndrome coronavirus Indoor and Built Environment $26,514$. doi:10.1177/1420326X16631596. 